\title{
El sistema Ctesiphonte. Evolución de la estructura catenaria
}

\author{
The Ctesiphont system. Evolution of the catenary structure
}

\author{
$\underline{\text { P. Rabasco }}^{(*)}$
}

\section{RESUMEN}

La complicada situación de la España de los años de autarquía (1940-55), llevó a que algunos arquitectos propusieran soluciones sumamente económicas y de rápida construcción para tratar de solucionar el grave problema de la falta de vivienda. Entre esas propuestas Ilama especialmente la atención el sistema Ctesiphonte, basado en una catenaria estructural como único elemento que define el espacio. La solución se extenderá para otras funciones como la agropecuaria, pero igualmente llegará a utilizarse para edificios de uso social o religiososos.

$509-2$

Palabras Clave: vivienda, catenaria, ctesiphonte, arquitectura social, estructura.

En el año 1947, el arquitecto finlandés afincado en los Estados Unidos, Eeron Saarinen, diseñaba junto al ingeniero de origen alemán Hannskarl Bandel (1), el Jefferson National Expansion Memorial, en la ciudad de St. Louis, en el estado de Missouri. Nacía así uno de los símbolos de la nueva arquitectura americana ${ }^{1}$. Pero de una manera un tanto irónica, esta estructura no estaba diseñada por un arquitecto americano, y ni siquiera se trataba de un edificio. Era una expresión de alta tecnología de la construcción aplicable a la arquitectura, tal y como se demostró en la propia carrera de los Saarinen, pero también a la ingeniera y al arte. En unas fechas realmente convulsas, recién terminada la segunda guerra mundial, los Estados Unidos empezaban a tomar conciencia de su

\section{SUMMARY}

The difficult situation in Spain during the autarchy years (1940-55) led some architects to propose extremely economic and rapid solutions in order to solve the serious problem generated by the lack of housing. One of these proposals, and of particular interest, is the Ctesiphon system, based on a catenary structure as the only element that defines the space. This solution was to be used later for agricultural, social and religious purposes.

Keywords: housing, catenary, ctesiphon, social architecture, structure.

papel en un nuevo orden mundial, y la arquitectura intentaba componer un nuevo lenguaje. Se trataba de adaptar las enseñanzas de los grandes maestros del Movimiento Moderno a una nueva realidad económica y social que no siempre posibilitaría los procesos ciertamente utópicos formulados en las décadas anteriores desde el viejo continente. (Figura 1)

El gran monumento de St. Louis, edificado en memoria de la expansión territorial norteamericana, no era otra cosa que una estructura formada por una triple catenaria invertida que constituía una sección triangular para conseguir una altura de $192 \mathrm{~m}$. Este gran despliegue de la tecnología, a otra escala tanto real como simbólica, estaba ya muy presente en Europa 
1. Jefferson National Expansion Memorial. Eeron Saarinen (1947).

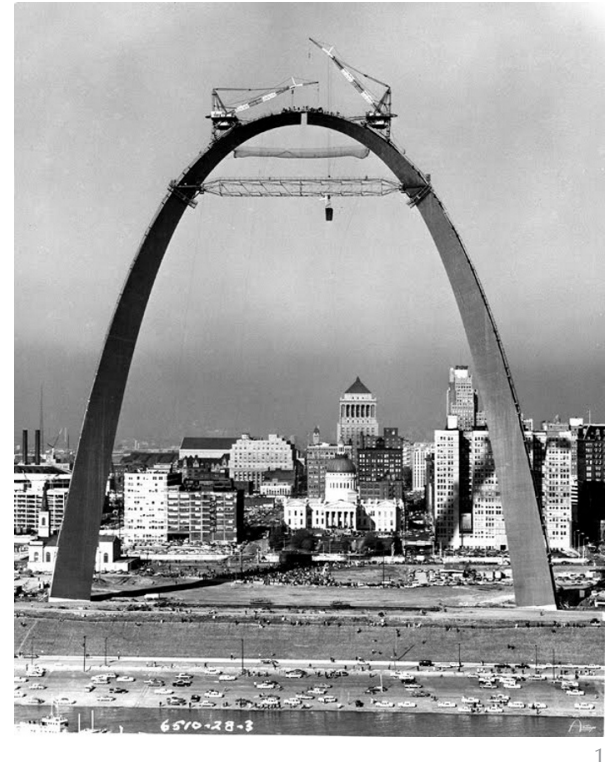

en la ingeniería de puentes, de uso militar o en la aviación civil, pero contrastaba en esas mismas fechas con formas similares que a partir de una catenaria invertida describían una estructura autoportante creando un espacio habitable. Fue en 1944 cuando estas formas catenarias aparecieron en el sur de Inglaterra, dando cobijo a miles de soldados que se preparaban para desembarcar en Normandía (2). A partir de esos momentos, una forma que había estado asociada a la arquitectura de funciones singulares o altamente tecnificadas, pasa a ser considerada una forma aceptable de arquitectura económica y de rápida construcción, normalmente asociada a entornos y espacios de usos secundarios (almacenes, establos...) pero que en el caso español llegará a conformarse como un ejemplo extremo de vivienda mínima.

A comienzo de la década de los 50 aparece en el panorama de la arquitectura española la necesidad de buscar nuevas tipologías que solucionen problemas concretos de una forma rápida y económica. Problemas que parecían concretarse en el diseño y planificación de vi- viendas de carácter social. El proceso, que busca adaptar las poco comprensibles pretensiones políticas a las escasas posibilidades tecnológicas desarrollables en una España recién salida del período de autarquía, es quizás el ejemplo más claro de un período donde la arquitectura, extremadamente dirigida e impuesta, choca con sus propias pretensiones, convirtiendo el resultado en una suerte de intentos que se sitúan en el mismo límite de la arquitectura habitable. Rafael de La Hoz, en su momento, analizó la situación de una manera muy acertada ${ }^{2}$.

Entre estas nuevas formas Ilama especialmente la atención la presencia de la tipología Ctesiphonte, una forma que fue aplicada de manera sorprendente en edificios con finalidades tan dispares como la industrial, la vivienda o la religiosa, en una concentración de funciones que marcaron realmente un ejemplo sin precedentes en la historia de nuestra arquitectura (3). Lo más destacable no es sin embargo que una tipología adopte funciones tan distintas, sino el hecho de resumir y acoger en una sola forma tendencias tipológicas que parecían inamovibles tanto en sus aspectos culturales, sociales o políticos (4).

La construcción tipo Ctesiphonte se desarrolla en España entre 1950 y 1965, en proyectos llevados a cabo por arquitectos como Alejandro de la Sota, Carlos de Miguel, José L. Picardo, Fernando Moreno Barberá o Rafael de La-Hoz. Tras este breve período de quince años, tiempo donde parece extenderse en un afán experimental sin precedentes, esta estructura desaparece y no vuelve a desarrollarse, al menos de la misma manera.

El desarrollo de esta forma de catenaria invertida y la adopción del nombre de Ctesiphonte por la cual la conocemos, parte del conocido arco que marca la entrada al palacio que lleva este mismo nombre, sede de la capital de los imperios parto (170 a 226 d.c.) y persa ( 226 a 650 d.c.). El palacio se encuentra en Salman Pak, en las cercanías de la ciudad de Bagdad.

${ }^{1}$ El Jefferson National Expansion Memorial, se ordena construir por; Executive Order 7523 de 21 de diciembre de 1935 bajo la responsabilidad del National Park Service.

2 "El conflicto que como arquitectos hemos de solucionar estriba en la reducida superficie que disponemos para desarrollar tal programa. Stratemann lo resuelve con un mínimo de 83,28 $\mathrm{m}^{2}$ construidos. La UIOF aconseja 75,70 $\mathrm{m}^{2}$ útiles. Sin embargo, el acuerdo 22-1-59 Arrese-Solís establece, respectivamente, $50 \mathrm{~m}^{2}$ y $38 \mathrm{~m}^{2}$ como máximo de dichas superficies; es decir, nuestras viviendas sociales deben desarrollarse en superficies que oscilen del 50 al 58 por cien de los límites lógicos.

Por otra parte, el coste unitario de la construcción de una vivienda de programa fijo es más elevado a medida que disminuye la superficie en que se desarrolla. La razón es obvia; por más que se comprima la vivienda, sus elementos caros, instalaciones de todo tipo, carpintería, etc.- permanecen invariables.

Por el contrario, las viviendas sociales hay que resolverlas en el límite más desfavorable del erróneo criterio opuesto, heredado por el MV del antiguo INV "cuando una vivienda disminuye de superficie, el coste de metro cuadrado lo hace proporcionalmente. Aceptando como real el valor oficial que corresponde a $76 \mathrm{~m}^{2}$, encontramos que el asignado a las viviendas de $50 \mathrm{~m}^{2}$ es un 50,5\% del que realmente debe tener. El problema que la vivienda social plantea puede resumirse en construir una vivienda en la mitad de superficie universalmente considerada mínima, con la mitad de presupuesto que realmente se precisa". De la Hoz, R.: "La vivienda social". Arquitectura, n 39, (1962). Este mismo texto lo incluye el autor en la Memoria para la construcción de 520 viviendas tipo social para la Obra Social Cordobesa Huertos Familiares, Córdoba Junio de 1959. Archivo del ministerio de la Vivienda, Delegación de Córdoba. 
Su curiosa forma y enormes proporciones han pasado a la historia de la arquitectura como el arco construido en ladrillo que alcanza mayores proporciones. Su traza de catenaria se describe a partir de una amplia base con siete metros de sección que va disminuyendo de grosor según va adquiriendo altura, hasta presentar en su punto más alto una mínima anchura, dando como resultado la sensación de fragilidad que sugiere su imagen. A pesar de esta sensación y los pequeños ladrillos utilizados, se ha mostrado con el paso de los siglos como una estructura sumamente robusta. (Figura 2)

Esta tipología se verá continuada en el tiempo, partiendo de tradiciones y usos indígenas sin que se registren influencias o modelos extranjeros directos en esta región. En otros edificios, como la iglesia de Ctesiphonte, construida entorno al año 600, vemos igualmente un desarrollo de esta forma donde desde la cabecera del templo arranca una catenaria que conforma el espacio (5). Los materiales son los mismos que observamos en toda la zona, básicamente ladrillo de adobe, a causa de la falta de piedra y los abundantes yacimientos de arcilla.

El estudio y desarrollo de la catenaria como elemento arquitectónico ya en la época contemporánea aparece fuertemente unido a la obra y las investigaciones de Antoni Gaudí. Estas formas las vemos aplicadas en obras como la desaparecida Cascada de la Casa Vicens, en la Casa Milá, en el Colegio de las Teresianas, en la Finca Güell, el Palau Güell, en Bellesguard o en la Sagrada Familia. Pero su uso se vuelve especialmente trascendente y rotundo en la bohardilla de la Casa Batlló, a base de un sistema de repetición de arcadas en catenaria realizadas en ladrillo.

Pero como bien ha señalado Joseph GómezSerrano, Gaudí no desarrolló por si solo las aplicaciones arquitectónicas de esta forma, algunos estudios venían profundizando en este sentido durante todo el siglo XIX. Autores como Gabriel Lamé, Poncelet, Kart Culman, Luigi Cremona, Scheffler o Carl Ritter habían dejado toda una serie de estudios a los que el propio Gaudí llegaría a través del conocimiento de la obra de Scheffler (6).

Igualmente, hemos de tener en cuenta la aplicación de esta forma por parte de Robert Maillart en muchas de sus obras, y especialmente en la influencia que tuvo en Félix Candela, autor que desarrolla estas mismas formas en la década de los 50:

"Le conocí en Space, Time and Architecture de Giedion, y conseguí después el libro de Max Hill, con su inestimable colección de ensayos publicados por Maillart. Devoré sus atículos Diseño y Cálculo del Hormigón Armado, en el que diferencia claramente el significado de

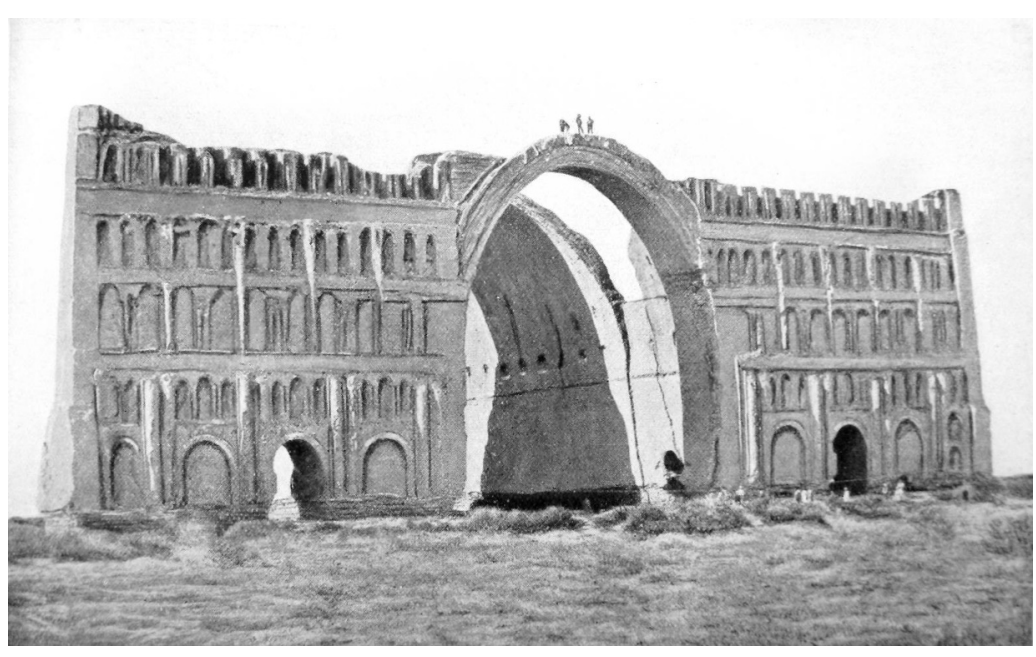

ambos términos tratando de evitar la, más que semántica, confusión que prevalece, sobre todo en los países de habla inglesa, entre diseño y cálculo; El Ingeniero y las Autoridades, que expresa su posición respecto al establecimiento, y Masa y Calidad en las Estructuras de Hormigón Armado" (7)

El hecho es que en torno a ese año de 1950 se da en España la estructura de catenaria invertida como definidora del espacio. El arquitecto Rafael de la Hoz, uno de los nombres más importantes en el desarrollo de la vivienda social en la España del franquismo, trató de rastrear esta tipología e igualmente afirmó que su primera aplicación se produce al Sur de Inglaterra pocas semanas antes del día D, como construcción destinada a albergar al gran número de tropas allí concentrado (2). Al terminar la Guerra se siguió experimentando con este nuevo sistema; "Posteriormente, a la terminación de la guerra, se construyó por el Ministerio de Obras Públicas de la Gran Bretaña, en el campo de pruebas del norte de Londres, un edificio Ctesiphon de 18,3 m. de luz por $61 \mathrm{~m}$. de longitud." Reafael de La Hoz comentaba en la descripción de esta tipología que "la idea del Ctesiphon moderno

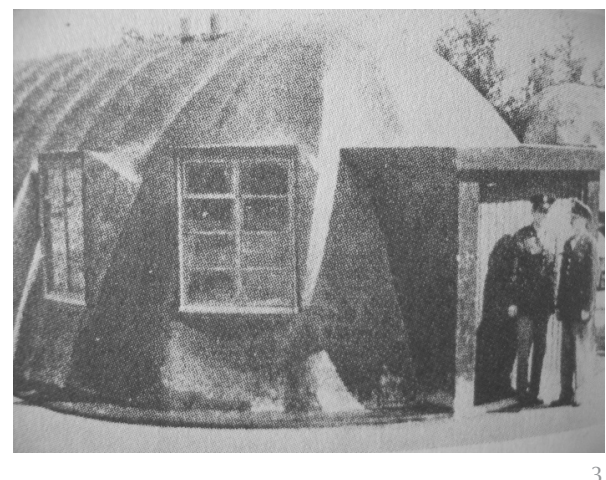

tuvo su origen en la contemplación del arco de Ctesiphon. El primer arco ondulado fue construido en 1941, denominándolo, con bastante propiedad, el moderno Ctesiphon. Su luz fue de 6,1 m., y el espesor de la lámina de 3,1 cm., no conteniendo ninguna clase de refuerzos." (9). (Figura 3) El mismo Félix Candela señalaba en 
4. Hangares de Orly. Eugene Freyssinet (1916-1924).

5. Les Halles Centrales de Boulinguin, Reims. Emile Maigrot (192728).

6. Dibujo de Alejandro de la Sota. Publicado en 1951 en el BGDA.
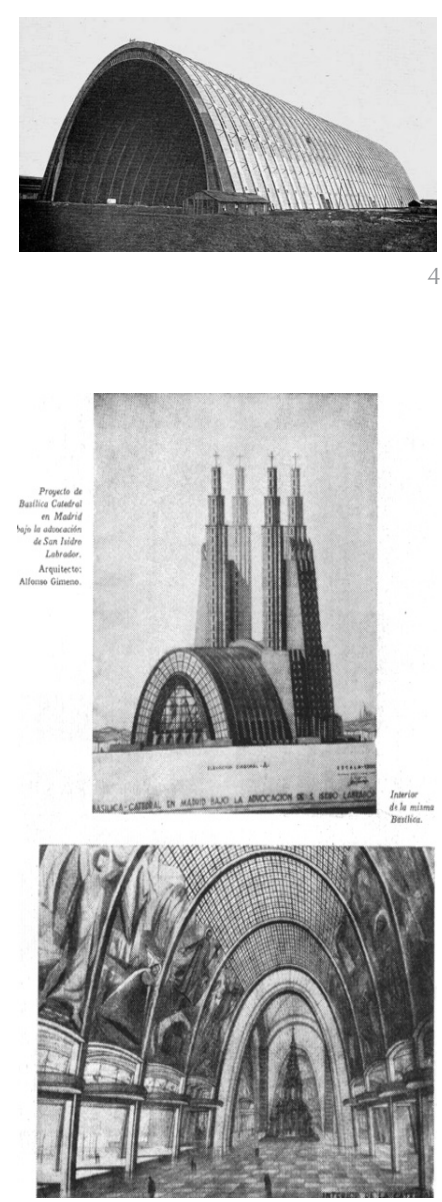

7. Proyecto de Basílica Catedral en Madrid. Alfonso Gimeno (1950).

8. Iglesia de Campamento, San Roque. (Cádiz)

3 Para ver la evolución de esta problemática en los años inmediatamente anteriores ver: Sambricio, C.: "La ingeniería en las revistas españolas de arquitectura: 1920-1936", en Informes de la Construcción, vol. 60, n 510, 2008.
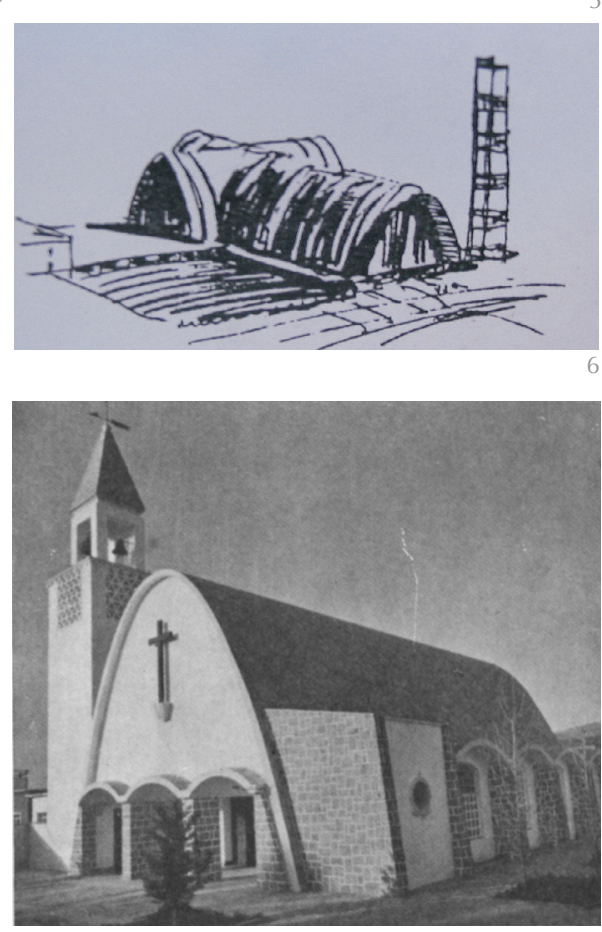

referencia a los comienzos de esta estructura que él mismo realizará a partir de 1950 que "Esto fue copiando un sistema que estaban haciendo en Inglaterra entonces; yo copiaba todo lo que podía" (10). La tipología la va a desarrollar el ejército norteamericano en estas circunstancias y la utilizará tanto como edificio de almacenamiento, como vivienda de altos cargos militares. Pero ya se habían producido algunos ejemplos de esta forma

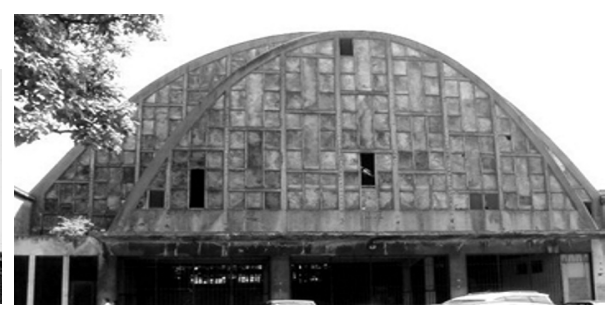

como elemento único estructural que define el espacio, en construcciones de tipo industrial como los Hangares en Orly de Eugene Freyssinet, realizados entre 1916 y 1924. Freyssinet ya realizó estas estructuras durante el segundo período de tiempo en que trabajó como ingeniero militar para el ejército francés entre 1914-18. (8). Igualmente lo aplica en estructuras abiertas como en el Tonneins Bridge, realizado entre 1919-22 (11). De la misma manera, otra obra muy interesante en este mismo sentido es Les Halles Centrales de Boulinguin en Reims, obra firmada por el arquitecto Emile Maigrot en 1927-28 bajo las propuestas del propio Freyssinet, (Figuras 4 y 5$),(12,13)^{3}$.

Este sistema de membrana continua nace en sus primeros momentos como un concepto mixto, aplicable tanto a vivienda como a almacén aunque, cuando concluye el conflicto se potencia su uso industrial, hasta que se rescata la posibilidad de vivienda en circunstancias también extremas como las que se daban en la España de los 50. Habría que recordar que durante los primeros años de la década de los cincuenta aparecían en prensa especializada detalles de la construcción de prototipos siguiendo técnicas similares aunque aplicadas en estructuras de grandes dimensiones.

El período clave para el desarrollo de esta tipología surge en el momento en que, esta forma se considera como elemento suficientemente despegado de la semántica del ingeniero como para definir conceptos arquitectónicos y tipológicos tan importantes para la propia historia de la arquitectura como lo son la vivienda o el templo religioso.

En España ese momento se da en 1951, cuando de manera diferente, una serie de arquitectos parecen asumir una nueva y poderosa función para esta tipología formal.

Por un lado, Alejandro de la Sota publicaba en el Boletín de Información de la Dirección General de Arquitectura (BIDGA), un artículo sobre el estado de la crítica arquitectónica (14). Se trataba de respetar nuevas formas y tipologías que por su formalidad pudieran sufrir rechazo por parte de la crítica más tradicional. Este artículo aparecía ilustrado con unos dibujos a mano alzada donde el arquitecto mostraba ejemplos de estas nuevas arquitecturas, brillantes e incomprendidas. Entre los dibujos de estas formas novedosas e innovadoras aparecía una obra de arquitectura religiosa que utiliza la tipología Ctesiphonte, a modo de dos naves que se cruzan para conformar la cruz latina, todo resumido en una estructura de catenaria que arranca desde el nivel del suelo. Una idea similar, dentro del ambiente ambiguo que prevaleció durante la I Bienal de Arte Hispanoamericano, podemos contemplarla en el proyecto de Alfonso Gimeno para la Catedral bajo la advocación de San Isidro Labrador, donde encontramos desfasado el mismo tema propuesto e incluso las referencias excesivamente claras a la Sagrada Familia de Gaudí, pero que resulta muy moderno en la concepción espacial a través de una serie de catenarias que muestran además un arriesgado planteamiento en la iluminación cenital del espacio $(15,16)$. Esta misma concepción a otra escala la veríamos unos años más tarde en la Iglesia de Valdecañas, realizada por el arquitecto Fernando de Urrutia Usaola (17), por encargo del Instituto Nacional de Colonización algunos años después, en 1958, o en la iglesia del poblado de origen militar de Campamento (Cádiz), obra de Francisco Echenegui (18). (Figuras 6, 7 y 8) 


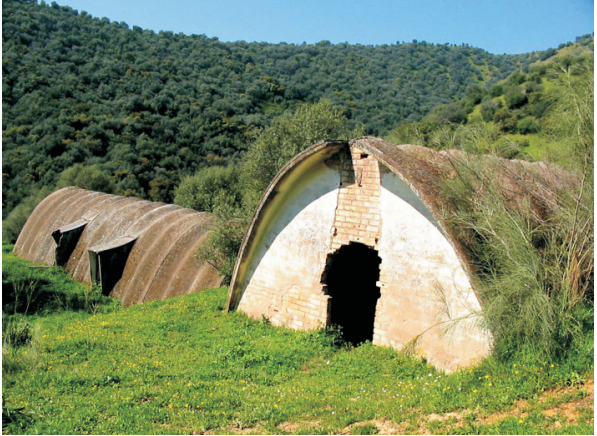

Se trata de un acercamiento conceptual a una serie de formas que se desarrollarían en los siguientes años, perdiendo ese concepto de arquitectura de calidad por el que apostaba De la Sota. (Figura, 9)

Carlos de Miguel y José L. Picardo la aplicaron en la construcción de unas naves de carácter industrial en el Centro Cívico para la Cofradía de Pescadores en Altea ${ }^{4}$. En este proyecto, la pareja de arquitectos colocaban un total de nueve construcciones utilizando el sistema Ctesiphonte, y aunque se centra en una serie de funciones de carácter más ligado a lo industrial, las proporciones se asemejan a los proyectos sociales que veremos a continuación. En este caso, los arquitectos proyectan nueve estructuras catenarias: dos como almacenes del Centro Cívico, cuatro depósitos para efectos navales y tres para depositar las redes que se sitúa a pie de embarcadero. Éstos se constituyen como construcciones auxiliares, pero realmente se incorporan correctamente al sentido general del proyecto. (Figura 10)

Pocos meses después, el influyente arquitecto Fernando Moreno Barberá publica en la revista Informes de la Construcción un proyecto de viviendas unifamiliares siguiendo el sistema Ctesiphonte. (Figuras 11 y 12) En esta ocasión desarrolló la tipología como residencia vacacional (19). En este proyecto, Moreno Barberá plantea cuatro puntos en los que se apoya para defender las ventajas de utilizar este sistema constructivo, ventajas que van desde conceptos puramente técnicos hasta algunos de tipo propagandístico y político, en un lenguaje de índole maquinista que se extiende en la España de los 50:

$1^{\circ}$. Los materiales que se emplean son de la mejor calidad, análogos a los de las viviendas de alto precio.

$2^{\circ}$. Por su especial sistema de construcción, las viviendas reúnen un buen aislamiento térmico, contando también con el debido confort como consecuencia de su estudiada distribución, e incluso su forma (empleada hoy en las ciudades atómicas de EE. UU.) es muy apropiada para resistir efectos de explosiones atómicas.
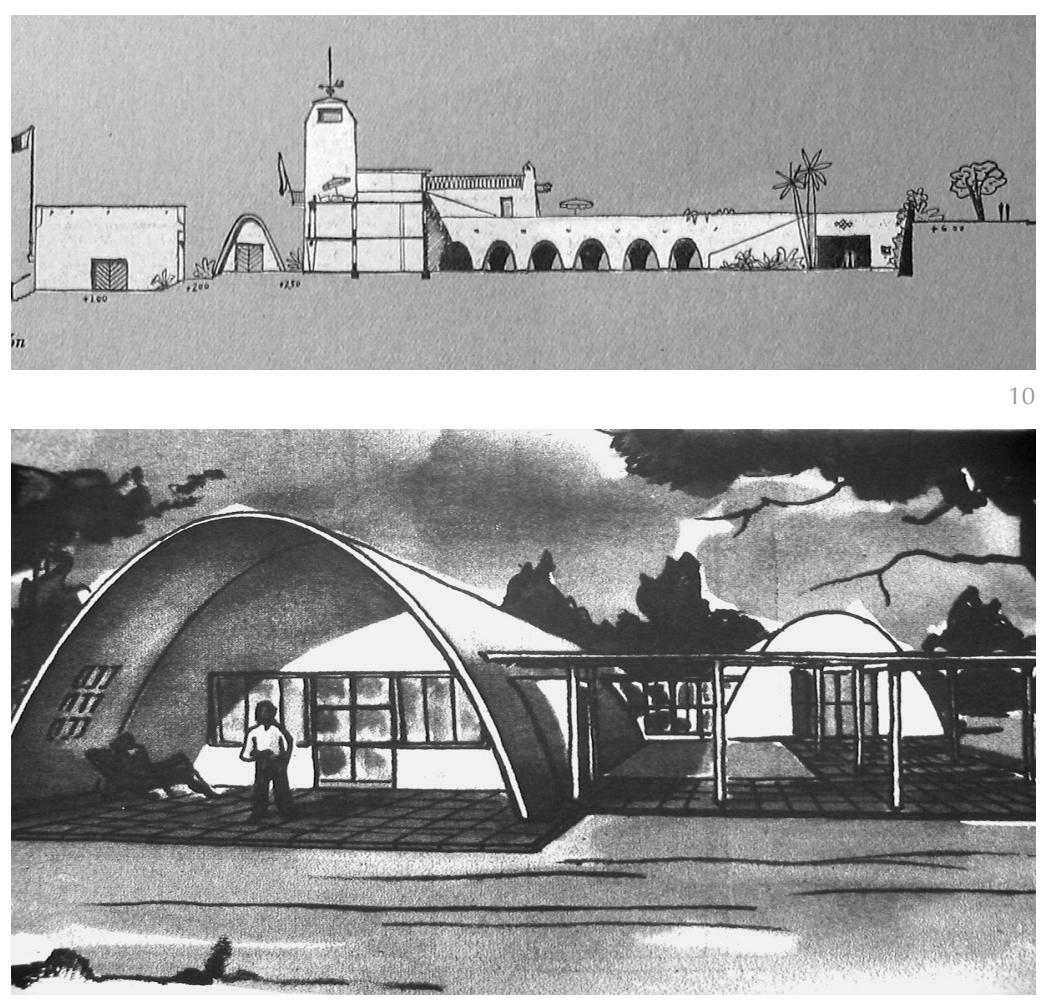

3\%. Se eliminan en casi su totalidad los materiales hoy escasos o caros en España: madera de cubierta y hierro en forjados, herrajes y clavazón. El gasto de cemento es inferior por metro cuadrado al que requeriría una construcción normal de ladrillo y mortero de cemento.

4. El especial sistema de construcción requiere un mínimo de horas de trabajo, base de su pequeño coste, y asimismo la intervención de un solo oficio -albañileríapara la total construcción de la estructura.

En el punto primero el arquitecto presenta ya la estrategia económica como elemento vital, pero no la muestra como un valor propio sino como un valor comparativo de contraste hacia otro tipo de viviendas, y más específicamente hacia otras clases sociales.

Resulta realmente curioso observar cómo posteriromente trata de establecer las cualidades físicas no perceptibles visualmente de la vivienda, pero igualmente lo hace en una escala cuando menos aclaratoria de la situación; nos habla de la temperatura, el confort y, conjuntamente a estas cualidades, la define como "apropiada para resistir efectos de explosiones atómicas" (19), aunque este punto se presenta como una simple anécdota, contiene un mensaje importante pues, pone en relación el proyecto con la tecnología desarrollada en los EEUU; es aquí donde el arquitecto pretende llegar para enlazar con otra tendencia muy común en la actualidad de las revistas de arquitectura
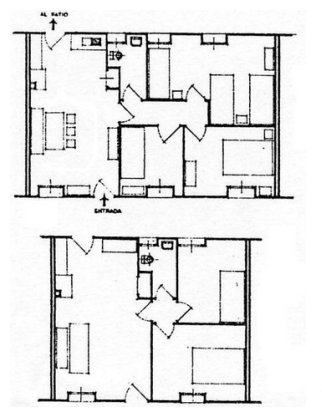

12

9. Estructuras agropecuarias en las cercanías de Córdoba.

10. Centro Cívico para Cofradía de pescadores. Altea. Carlos de Miguel y José L. Picardo (1951).

11. Proyecto de Fernando Moreno Barberá (1951).

12. Plantas del conjunto residencial. Moreno Barberá (1951).

${ }^{4}$ De Miguel, C., Picardo, J. L. "Centro Cívico para la Cofradía de Pescadores de Altea-Alicante". Revista Nacional de Arquitectura, $\mathrm{n}^{\circ}$ 115, (1951), pp. 36-38. Igualmente, en 1952 se publica; "Arcos de madera laminada". Informes de la Construcción, (1952), trascripción de un artículo publicado en The National Builder titulado "Laminated Timber Arches", que muestra el desarrollo del tipo Ctesiphonte como gran puerta de presentación al Festival Británico que en ese año se celebró junto a la estación de Waterloo. 
desde mediados de los años 40 pues, de cara al público, el sistema de apertura de la arquitectura española se muestra hacia el otro lado del océano como un referente tecnológico menos implicado en las tensiones políticas heredadas del conflicto.

Seguidamente, los puntos tercero y cuarto señalados en el texto, se convierten en un perfecto resumen de dos planteamientos muy comunes en estas fechas pero que fueron polémicos $y$, en ocasiones, contradictorios: el abaratamiento de los materiales y, lo que se presenta más discutible, la reducción de la mano de obra. El problema de esta reducción de la mano de obra se mezclaba con otro, el correspondiente inconveniente del aumento de paro en el sector de la construcción. Igualmente esto podía provocar una escasez de estos profesionales especializados y el descontento general en el sector. Conocemos que la cuestión es compleja pues la necesidad imperiosa de construcción de viviendas de bajo precio era de suma importancia para el nuevo régimen, pero cuando ya a partir de 1954 se necesita gran cantidad de mano de obra para proyectos de mayor envergadura, se tratará de corregir esta tendencia, que pasará a equilibrarse ante la necesidad de construcciones en altura que abaraten el costo de urbanización y el del metro cuadrado, y no el de la mano de obra.

No podemos olvidar que el proyecto aquí presentado se muestra como experimental, sin hablar de localización concreta, y como tal se refuerza esta orientación a través de la explicación de una serie de pruebas de resistencia constructiva que se detallarán en el proyecto.

En cuanto a la descripción formal del proyecto hemos de partir de la cualidad constructiva que lo define, es decir, del hecho de que todos los elementos de la vivienda son fabricados en serie. El sistema constructivo se mostró bastante simple y rápido en su ejecución; se trataba en primer lugar de elegir un terreno óptimo y orientar correctamente la estructura en la medida de lo posible. Seguidamente se colocaba en el suelo una plataforma que se convierte en la base de la vivienda, se montan una serie de cimbras a lo largo de esta base que conforman el armazón y que servirá como molde-soporte de una serie de capas de cemento y de aislantes que una vez secas permiten retirar estas cimbras y dejar fijada la estructura definitiva de la vivienda. Las cimbras podían ser de diversos materiales; tubos de hierro, madera...la elección de estos vendrá por el precio del material en el momento de la ejecución del proyecto ${ }^{5}$.

En referencia a la forma descrita por este arco habría que establecer algunos paráme- tros interesantes que afectan al análisis de la obra. Por un lado se trata de una forma despegada de la tradición arquitectónica para su uso como vivienda. Se separa igualmente de los planteamientos más funcionales por la problemática en el aprovechamiento del espacio que puede presentar, tanto a nivel de mobiliario, que queda muy determinado por la forma curva de las paredes, como en la de los movimientos de los habitantes de la casa que quedan también, de alguna forma, más restringidos por las diferentes alturas. Igualmente se potencia el significado orgánico del hogar, que cambia el sentido de centralidad a partir de ángulos, pasando a una interpretación del espacio radial, que hace que una persona que se encuentre sentada en una de las habitaciones este siempre cerca del centro insinuado de la concepción tridimensional del espacio.

Si la relación interior/exterior del proyecto se presenta cuando menos interesante, la relación entre diversas estructuras plantea igualmente tensiones que preocupan a Moreno Barberá y a las cuales dedica especial atención, tanto en la redacción como en las ilustraciones explicativas de su proyecto.

El primer problema que se presenta es el de potenciar la vivienda aislada o, por el contrario, optar por una sucesión de elementos habitacionales compartiendo muro de medianera; el arquitecto parece decantarse por este segundo, por economía en el uso de materiales y seguramente por la facilidad de urbanización del terreno aunque muestra la posibilidad de la construcción de residencias aisladas con este sistema.

Y aunque queda claro que el uso de estas viviendas se proyecta como segunda residencia y nunca como vivienda social, la distribución en planta de los tipos menores de $39,7 \mathrm{~m}^{2}, 42 \mathrm{~m}^{2}$ y $51,6 \mathrm{~m}^{2}$ respectivamente, plantea soluciones cercanas a las proporciones de lo social, y más concretamente en la elaboración de un mobiliario adaptado a su reducido tamaño y especial forma en el alzado. Afirmaciones como "todos los elementos complementarios pueden fabricarse en serie" (19), "cuando sea necesario, por medio de camas plegables y bancos-camas, como se detalla en los plano y será objeto de proyecto especial." o "Dos camas abatibles alojadas en nichos de fábrica, y en caso de necesidad el banco-arcón, sirven de dormitorio de hijos." (19), nos hablan de esa necesidad que posteriormente planteará Rafael de La-Hoz en el proyecto de Viviendas Ultrabaratas de Palma del Río (Córdoba, 1953) y que aplicará, al menos en el proyecto, en las viviendas del Grupo Francisco Solano de Montilla tres años más tarde, y que ahora 
se propone como una opción novedosa (3) (20).

Carlos de Miguel proyectaría algo más tarde un conjunto de viviendas para pescadores en este mismo sentido, pero con unas dimensiones y un sentido aun lejano a la arquitectura mínima pretendida por Rafael de la Hoz a partir de estas formas. En el Perellonet, en Valencia, dejaría un conjunto de 27 viviendas con unas superficies que oscilarían entre los $54 \mathrm{~m}^{2}$ y los $42 \mathrm{~m}^{2}$ (21). (Figura 13)

Pero en 1951, Rafael de La-Hoz y J. Ma . García de Paredes utilizarán esta tipología como paradigma mínimo de vivienda de tipo social al que denominaría vivienda ultrabarata. El número 135 de la Revista Nacional de Arquitectura, publicado en 1953, dedicaría una especial atención al proyecto en su artículo más extenso (22). El proyecto se debió comenzar en torno a 1951 puesto que en el texto publicado en la Revista Nacional de Arquitectura se hace referencia a que los arquitectos llevaban año y medio experimentando con estas formas. En el proyecto vemos la aportación más radical que hasta esas fechas se había realizado a la búsqueda y desarrollo de soluciones para solventar el problema de la vivienda. El tiempo se encargaría de no hacerlo tan radical, especialmente a partir del desarrollo de las tipologías de prefabricados en los albergues provisionales que se extienden por nuestras capitales en los primeros años de la década de los 60. (Figura 14)

Rafael de la Hoz tenía 30 años cuando realizó el proyecto de Palma del Río. El arquitecto se había titulado en 1950 en la Escuela Superior de Arquitectura de Madrid y de inmediato se trasladó a Córdoba donde su padre, el arquitecto Rafael de la Hoz Saldaña venía desarrollando su carrera profesional.

Será durante estos primeros años de producción donde debamos rastrear el significado de sus proyectos dedicados a la arquitectura social y en especial el que aquí nos interesa. Desde su salida de Madrid y hasta 1956, año en que recibe el Premio Nacional de Arquitectura junto a García de Paredes por la madrileña Residencia Aquinas en la Ciudad Universitaria, se moldea la personalidad como arquitecto y cobra sentido de coherencia el conjunto de su producción.

En las viviendas ultrabaratas que construye en Palma del Río (Córdoba), la superficie que ocupa cada vivienda se establece en 20 $\mathrm{m}^{2}$, número resultante del cálculo de la renta posible que podía pagar cada familia. El sistema lo venía empleando el arquitecto desde el año 1951 aunque el proyecto de Palma del Río se presenta como una definición de la
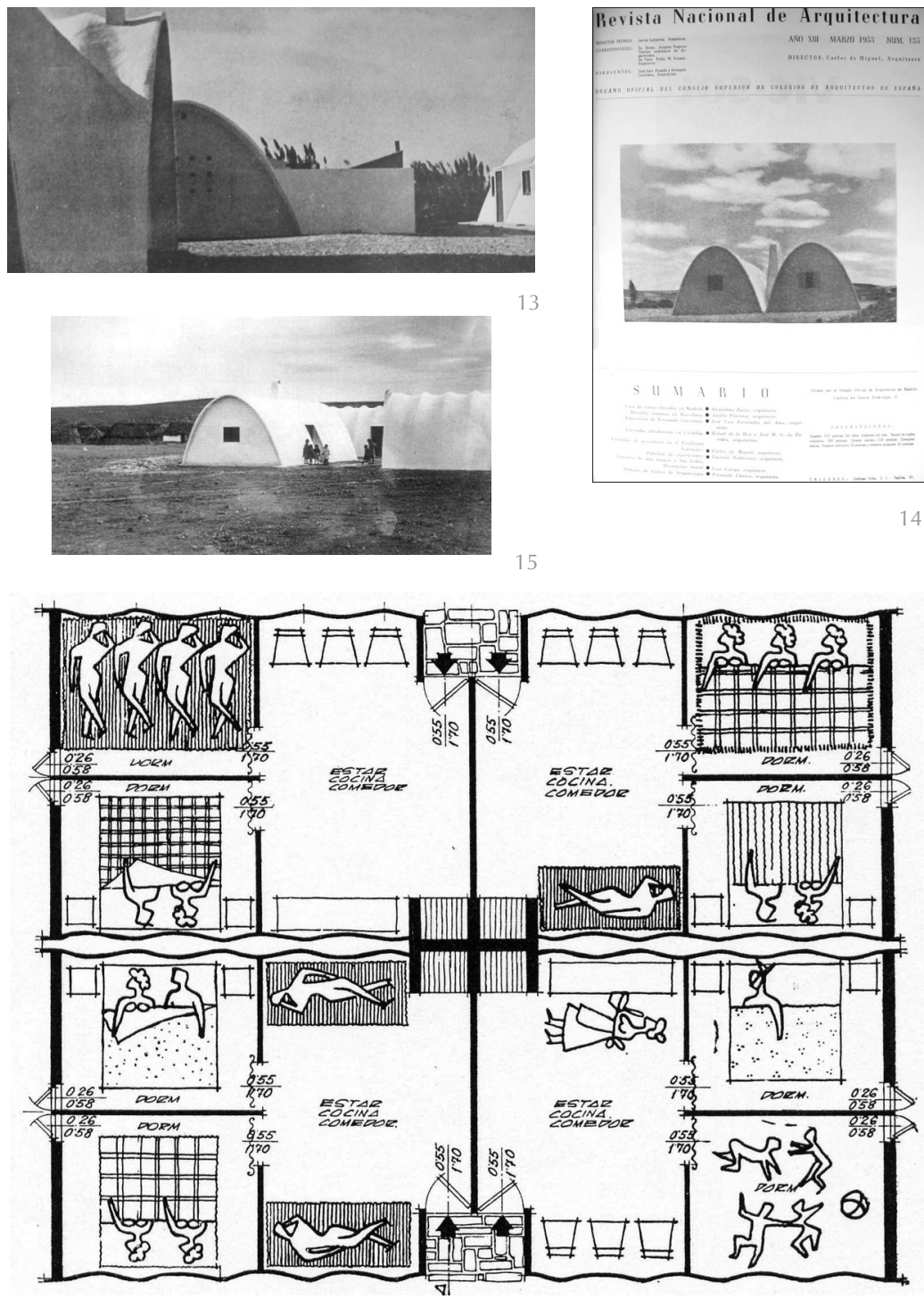

experiencia y de su aplicación a la vivienda de tipo ${ }^{6}$. (Figuras 15 y 16)

Las viviendas se disponen pareadas para formar un conjunto de cuatro con un pequeño espacio compartido que da entrada a cada vivienda. Ésta se compone de un espacio que hace las funciones de estar-cocina-comedor y dos dormitorios. La estancia principal tiene un hogar-chimenea y tan sólo presenta dos pequeños ventanucos en las habitaciones, con lo que la iluminación de la habitación principal se resuelve a través de la puerta de entrada, no quedando resuelto este punto ni el de la ventilación en el interior de la vivienda. El arquitecto se centró a la hora de la redacción del proyecto en cuestiones como la impermeabilidad, aislamiento térmico y las características técnicas tanto de la construcción del edificio como en su estabilidad ante presiones, viento, movimientos de tierra etc.

La habitabilidad se calcula con un número de seis habitantes por vivienda con lo que
13. Viviendas de pescadores en el Perellonet, Valencia. Carlos de Miguel (1953).

14. Portada interior $n^{\circ} 135$ de la Revista Nacional de Arquitectura (1953).

15. Viviendas ultrabaratas en Palma del Río. Rafael de la Hoz (1953).

16. Viviendas ultrabaratas. Plantas.

\footnotetext{
6 "Así, se ha realizado una sella experimental con sus cuatro viviendas de $3 \times 4 \times 5$, cuyo valor, dentro de su extrema modestia, es el de solucionar realmente el problema de la vivienda para quienes sólo disponen de 20 pesetas mensuales para esta necesidad vital, y únicamente con la aportación interesada del capital privada.". De la Hoz, R.;

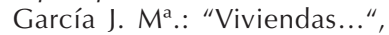
op. cit.
} 
17. Viviendas ultrabaratas. Rafael de la Hoz. Detalles de la construcción. resulta un número de $3,2 \mathrm{~m}^{2}$ por persona. Las cuestiones más estrictamente técnicas de uso de materiales fueron las siguientes según la expresaron los arquitectos: (Figuras 17, 18 y 19)

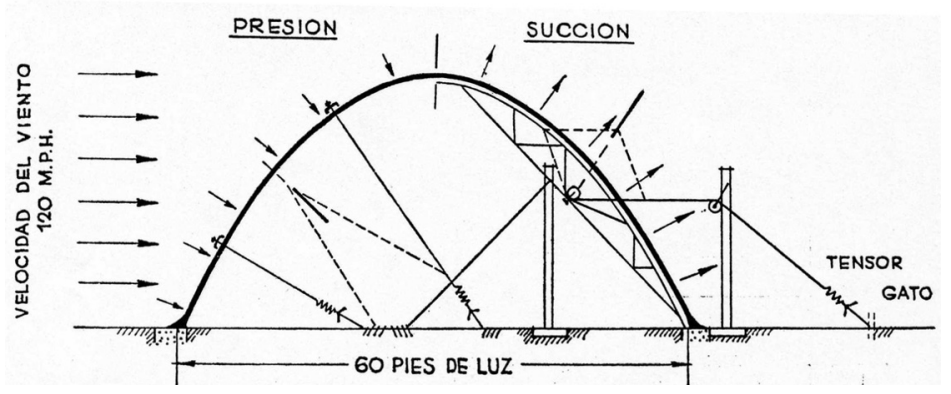

17

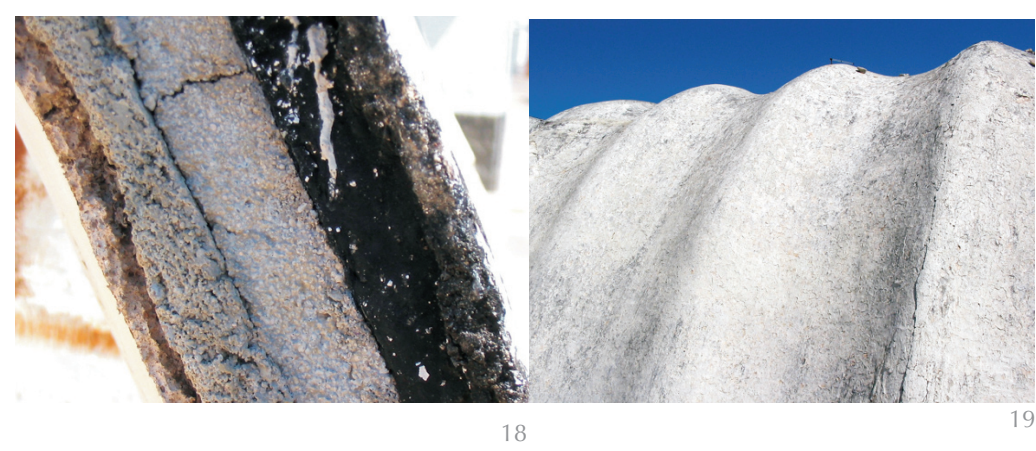

18. Viviendas ultrabaratas. Rafael de la Hoz. Detalles de la construcción.

19. Viviendas ultrabaratas. Rafael de la Hoz. Detalles de la construcción.
Impermeabilidad. Perfecta, dado que la membrana está comprimida en todos sus puntos; una tela metálica ha absorbido las retracciones de fraguado, y juntas de dilatación impiden tracciones por variación térmica.

Falla en los siguientes casos: Cuando la arena del mortero contiene más de un 3 por 100 de arcilla (la solución es lavarla). Cuando el fraguado del mortero ha sido demasiado rápido (hay que mantenerlo húmedo unos seis días. Cuando no trabajan bien las juntas de dilatación (hoy las empleadas, excelentes, del tipo piscina en cinc o plomo). Cuando sobrevienen asientos de la cimentación (conviene armarla con 4/8)

Aislamiento térmico. Ningún clima más duro en verano que el de Córdoba, y en esta provincia el de Palma del Río, con 68 grados al sol y 46 a la sombra. Pues bien: el resultado ha sido sorprendente. Es el tipo de construcción conocido más fresco en verano.

Las razones son las siguientes:

$1^{\text {a }}$. La superficie expuesta al sol es prácticamente la mínima posible.

$2^{a}$. El blanco de toda la superficie encalada actúa como aislante por reflexión, análogamente a los aislamientos por lámina de aluminio.

$3^{a}$. La gran conductividad térmica del hormigón hace que durante la noche se refri- gere por completo, no almacenando calor de un día para los siguientes (la técnica del attic fan americano).

En resumen, equivale a una chaqueta blanca.

Contra el frío. El interior de estas bóvedas las enlucimos con mortero de yeso y paja machacada en un grueso total de $2,5 \mathrm{~cm}$.

Esta capa es un freno a las pérdidas de calor interior, que se mantiene regularmente. Sin embargo, dadas las temperaturas relativamente moderadas de nuestro invierno, sin grandes continuidades en el frío, este aspecto deberá ser estudiado en otras regiones españolas.

La única dificultad que el sistema presenta en invierno es que la humedad interior se condensa en las ventanas por las noches.¡cómo se empañan los cristales!-, dificultad que se soluciona con el enlucido antes mencionado.

Características técnicas.

Una de las principales ventajas del hormigón es su propiedad de poder ser moldeado. Sin embargo, su utilización más frecuente, hasta la fecha, ha sido en formas semejantes a las adoptadas al utilizar en la construcción los materiales precedentes.

El hormigón se diferencia de la madera y del hierro en su falta de capacidad para resistir a la extensión, de donde se deduce que en las estructuras prismáticas reticulares de hormigón armado una gran cantidad del hormigón tenga una función resistente inútil y cumpla solamente a la de cubrición de hierro.

Resulta complejo buscar la razón de ser de este tipo de proyecto en el ámbito de la España que sale del período de autarquía. Ciertamente, la trascendencia como modelo habitacional fue mínima, pero el hecho de que tuviera su presencia en los medios especialistas de divulgación nos hace pensar en una cierta tendencia que, tanto en Rafael de La-Hoz como en otros arquitectos del momento era valorada como una serie de aportaciones técnicas que, desarrollándose fuera de nuestras fronteras podían aplicarse a los problemas específicos de nuestro país.

Y el caso es que en el espíritu general del texto notamos lo que posteriormente sería una constante en la postura de Rafael de la Hoz ante el problema de la vivienda. El arquitecto puede construir muy barato, estudiar los materiales más asequibles a la situación de nuestra industria de la construcción y las técnicas más económicas, pero también sabe que en ciertos momentos se está superando con creces el límite de habitabilidad de una forma innecesaria. Unos años después, Rafael de la Hoz se encontraría ante una problemáti- 
ca similar que cambia de escala. Ante la masiva e imperiosa necesidad de construcción de viviendas temporales, el arquitecto optará por soluciones acordes a una industria algo más desarrollada que posibilitaba la construcción en serie de elementos prefabricados, que permitían la construcción de grandes barriadas donde acoger a los desplazados de las zonas rurales. Personas que buscaban empleo y mejores condiciones de vida en las capitales de provincia. Así, entorno a los primeros años de la década de los 60, aparecerán grandes proyectos de urgencia social elaborados a partir de elementos prefabricados. En el caso de Rafael de la Hoz, en Córdoba en 1963, llevó a cabo la construcción de 3.592 albergues provisionales en lo que fue, según la prensa local cordobesa, el mayor conjunto de viviendas prefabricadas que se había hecho hasta el momento en España. El proyecto se realiza a través del Ministerio de la Vivienda en colaboración con la Obra Sindical del Hogar, en colaboración con el arquitecto Gerardo Olivares en lo que formaba en esos momentos una de las parejas profesionales más brillantes y fructíferas.

Y aunque lo hemos citado anteriormente, no podemos olvidar que otro arquitecto español en esas mismas fechas estaba trabajando de una manera insistente en estas mismas formas. Félix Candela hizo de estos "cascarones" uno de los símbolos de crecimiento de su propia identidad como arquitecto, y aunque tuvo que llevar a cabo su carrera en México por la suerte del exilio, es cuando menos curioso observar cómo en estas mismas fechas está muy cercano a las estructuras Ctesiphontes aquí desarrolladas.

Quizás la obra que se encuentre más sorprendentemente ligada a las propuestas españolas sea el conocido Laboratorio de Rayos Cósmicos, realizado en México D.F. en 1952, donde junto al arquitecto Jorge González Reyna lleva a cabo una obra que en esta ocasión presenta una estructura autoportante de arcos hiperbólicos paraboloides, pero construida siguiendo un sistema muy similar al visto hasta ahora y en la misma línea de las propuestas vistas anteriormente $(23,24)$. Es su aportación más conocida desde la estructura autoportante, pero no la primera. En 1950 había construido en la Escuela Experimental en Ciudad Victoria (Tamauripas, Méjico) una nave Ctesiphonte de 6 metros de altura. Candela se refiere de esta manera a dicha obra: "Lo primero que hicimos fue una obrita muy chiquita, como de 6 m., que está hecha sin armadura, sin hierro y sin cimbra, de encofrado. Entre medias se pone tela de saco y después, si se puede, que está medio difícil, se va aplicando el mortero de cemento, que es difícil que no se caiga; pero al final los albañiles mejicanos son muy
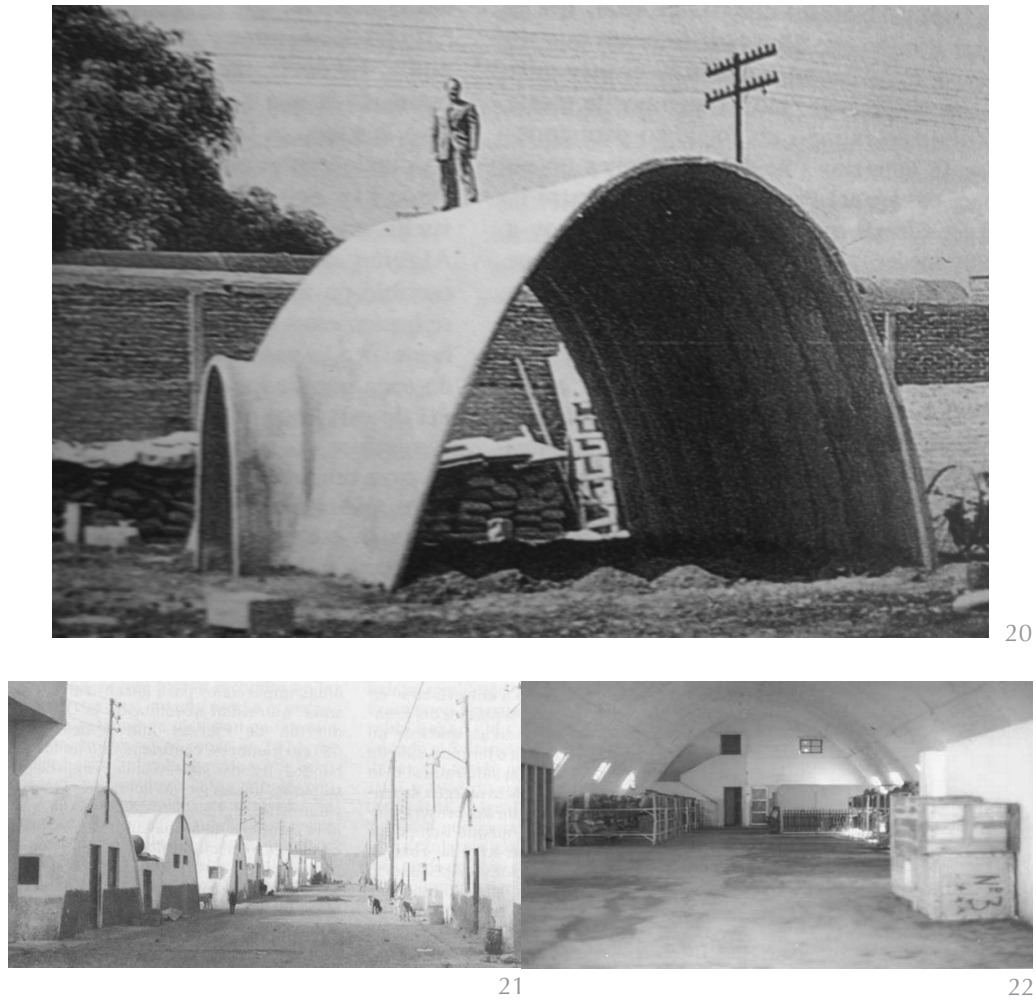

habilidosos y sonsiguieron que no se cayera. Luego quitamos las cimbras y ahí se quedó." (10). (Figura 20)

La catenaria invertida se convirtió a partir de estas fechas en una solución aunque no de una forma mayoritaria, si que fue salpicando buena parte del teritorio español para solucionar especialmente estructuras de uso agropecuario y de almacenaje.

En este sentido, cabría destacar la especial trascendencia de este concepto en los conjuntos residenciales que se llevan a cabo en Ifni y el Sahara españoles en los últimos años de control del territorio. En las ciudades de Sidi Ifni, El Aaiún o en Villa Cisneros aparecen viviendas y almacenes construidos con la estructura de una forma ciertamente naturalizada. El especial tratamiento de las construcciones en estas poblaciones se debe a un alto nivel de experimentación al amparo de las especiales condiciones que allí se dieron: climáticas, residenciales, tanto para nativos como para colonizadores, y también por lo alejado del territorio peninsular. Todo esto posibilitó la aparición de estructuras novedosas, de extrañas conjunciones donde la modernidad, un cierto orientalismo y lo imperioso de la función-símbolo condicionaron algunas obras ciertamente destacables en el ámbito de la arquitectura y del urbanismo (25). (Figuras 21 y 22)

Fueron utilizadas con más frecuencia en las zonas separadas de acuertelamiento, para viviendas o comedores de los soldados de la tropa, pero también se construyeron angares 
de grandes dimensiones en los aeródromos militares. Pasando de servir como edificios secundarios, a describir formas simbólicas, como en el Cuartel General de El Aaiún, donde la entrada queda estructurada mediante un amplio arco de catenaria. En ocasiones se plantean dentro de la planificación urbana llevada a cabo ya en la década de los 60, y otras veces aparece como edificio marginal, en un contexto temporal muy diferente al desarrollo anterior. Son numerosos los conjuntos de viviendas encadenadas que se construyen a partir de la estructura Ctesiphonte en Ifni y en todo el Sahara español, formas que en estas latitudes se visibilizan con mayor naturalidad que en la península pues se acomodan al normal desarrollo de una arquitectura vernácula donde las cubriciones suelen resolverse medianate cúpulas visibles desde la calle. Podemos afirmar que será aquí, en la región del Sahara Occidental, donde la catenaria invertida tendrá una trascendencia más que notable, siendo una forma que se adapatará a la tradición vernácula, al afán experimental de los arquitectos españoles que trabajaban en la zona, y a las especiales condiciones económicas, constructivas y sociales.

\section{BIBLIOGRAFÍA}

(1) Tedesko, A.: "Hannskarl Banden, 1925-1993)", en AA.VV.: Memorial Tributes: National Academy of Enginnering, vol. 8, (1996), pp. 17-19.

(2) Mallory, K.; Ottar, A.: Architecture of agresión. A history of military architecture in North West Europe 1900-1945. Ed. Architectural Press. Londres (1973).

(3) Rabasco, P.: "La vivienda mínima en Rafael de la Hoz. Ejemplos extremos", en AA.VV.: Modelos alemanes e italianos para España en los años de la posguerra. Editorial T6, Escuela Técnica Superior de Arquitectura Universidad de Navarra, Pamplona (2004).

(4) AA.VV.: Monográfico. Miguel Fisac (1913-2006), en Informes de la Construcción, vol. 58, $\mathrm{n}^{\circ} 503$.

(5) Krautheimer, R.: Arquitectura Paleocristiana y Bizantina. Cátedra. Madrid (1993), pp. 350-351.

(6) Gómez-Serrano, J.: "Arcos Catenarios", en Gaudí. La búsqueda de la forma. Espacio, geometría, estructura y construcción. Catálogo de Exposición, Dir. Daniel Giralt-Miracle, Ed. Ayuntamiento de Barcelona, Institut de Cultura y Lunwerg Editores S.A. Barcelona, (2002).

(7) Candela, F.: En defensa del formalismo y otros escritos, Xarait Ediciones. Bilbao, (1985).

(8) Candela, F.: En defensa del formalismo y otros escritos, Xarait Ediciones. Bilbao, (1985).

(9) De la Hoz, R.; García J. Ma.: "Viviendas ultrabaratas en Córdoba". Revista Nacional de Arquitectura, 135, (1953), p. 17.

(10) Candela, F.: Félix Candela. Arquitectura, no 303, p. 44.

(11) Rui-Wamba, J.; Fernández Alba, A.: Eugène Freyssinet. Un ingeniero revolucionario. Fundación Esteyco, Madrid, (2003).

(12) Prentiss, L.W.: "Techumbre de hormigón para un hangar de aviones de bombardeo". Informes de la Construcción, $\mathrm{n}^{\circ}$ 23, (1950).

(13) "Hangares de hormigón para bombardeos". Informes de la Construcción, (1955), p. 67.

(14) De la Sota, A.: "Crítica de Arquitectura". Boletín de Información de la Dirección General de Arquitectura. Volumen V, (1951), pp. 25-28.

(15) Gimeno, A.: "Proyecto de Basílica Catedral bajo la advocación de San Isidro Labrador". Cortijos y Rascacielos, $n^{\circ}$ 67-68, (1951)

(16) Del Real, P.: "Construcciones de América: La exposición Arquitectura actual de América y los discursos de representación", en AA.VV. Miradas cruzadas. Intercambio entre Latinoamérica y España en la Arquitectura española del siglo XX, T6 Ediciones, Pamplona (2008), pp. 187-194.

(17) Pérez, F.: "Las nuevas formas de colonización de la arquitectura de posguerra en la obra de Fernando de Urrutia Usaola: Arquitectura para Regiones Devastadas, los poblados hidroeléctricos y ciudades jardín en la periferia". Arquitectura, ciudad e ideología antiurbana. T6 Ediciones, Pamplona, (2002), pp. 159-167.

(18) "Iglesias de la Dirección General de Arquitectura". Revista Nacional de Arquitectura, n ${ }^{\circ} 73$, (1965), pp. 27-29.

(19) Moreno, F.: "Proyecto de viviendas unifamiliares". Informes de la Construcción, no 35, (1951).

(20) Sambricio, C.: « Rafael de la Hoz. Viviendas en Montilla, Córdoba (1957-1962)". Los Brillantes 50. 35 proyectos. T6 Ediciones, (2004), pp. 122-131.

(21) De Miguel, C.: "Viviendas de pescadores en el Perellonet (Valencia)". Revista Nacional de Arquitectura, $\mathrm{n}^{\circ} 13,(1953)$, p. 21.

(22) De la Hoz, R.; García J. Mª.: "Viviendas ultrabaratas en Córdoba". Revista Nacional de Arquitectura, no 135, (1953), p. 17.

(23) Candela, F.: Félix Candela. Idea Books, (1995).

(24) Richardson, S.: Félix Candela; Shell Builder. A Biography. Ed. Vance Bibliographies, (1989).

(25) Sahara Ifni. España en Paz. Conmemoración de los 25 años de paz. Madrid, (1965). 